\title{
Deterministic character of all-optical magnetization switching in GdFe-based ferrimagnetic alloys
}

\author{
L. Le Guyader, ${ }^{1, *}$ S. El Moussaoui, ${ }^{2, \dagger}$ M. Buzzi, ${ }^{2}$ M. Savoini,,${ }^{3, \ddagger}$ A. Tsukamoto, ${ }^{4}$ A. Itoh,${ }^{4}$ A. Kirilyuk,,${ }^{3}$ Th. Rasing, ${ }^{3}$ \\ F. Nolting, ${ }^{2}$ and A. V. Kimel ${ }^{3}$ \\ ${ }^{1}$ Helmholtz-Zentrum Berlin für Materialien und Energie GmbH, Albert-Einstein-Strasse 15, 12489 Berlin, Germany \\ and Swiss Light Source, Paul Scherrer Institut, CH-5232 PSI-Villigen, Switzerland \\ ${ }^{2}$ Swiss Light Source, Paul Scherrer Institut, CH-5232 PSI-Villigen, Switzerland \\ ${ }^{3}$ Radboud University Nijmegen, Institute for Molecules and Materials, 6525 AJ Nijmegen, The Netherlands \\ ${ }^{4}$ College of Science and Technology, Nihon University, 24-1 Narashinodai 7-chome, Funabashi-shi, Chiba 274-8501, Japan
}

(Received 2 December 2014; revised manuscript received 29 February 2016; published 1 April 2016)

\begin{abstract}
Using photoemission electron microscopy with x-ray magnetic circular dichroism as a contrast mechanism, new insights into the all-optical magnetization switching (AOS) phenomenon in GdFe-based rare-earth transitionmetal ferrimagnetic alloys are provided. From a sequence of static images taken after single linearly polarized laser pulse excitation, the repeatability of AOS can be quantified with a correlation coefficient. It is found that low coercivity enables thermally activated domain-wall motion, limiting in turn the repeatability of the switching. Time-resolved measurements of the magnetization dynamics reveal that while AOS occurs below and above the magnetization compensation temperature $T_{\mathrm{M}}$, it is not observed in GdFe samples where $T_{\mathrm{M}}$ is absent. Finally, AOS is experimentally demonstrated against an applied magnetic field of up to $180 \mathrm{mT}$.
\end{abstract}

DOI: 10.1103/PhysRevB.93.134402

\section{INTRODUCTION}

Controlling magnetism on the ultrashort time scale of sub-100 ps has become an important research subject, not only for the potential applications in novel high-density and high-speed magnetic recording technologies, but also for the unique opportunity to investigate magnetism on the fundamental time scales of the interactions between electrons, spins, and lattice [1]. The demonstration [2] of a rather unexpected ultrafast sub-ps demagnetization in a thin Ni film upon femtosecond laser excitation inspired a large number of subsequent studies [3]. Of particular importance was the surprising demonstration of a deterministic magnetization reversal by the sole action of a single 40 fs laser pulse in $\mathrm{GdFeCo}$ rare-earth-transition-metal (RE-TM) alloys [4]. The microscopic mechanism responsible for this phenomenon, now referred to as all-optical switching (AOS), is still a matter of debate. Element selective studies of ultrafast demagnetization in $\mathrm{GdFeCo}$ alloys led to the interpretation that $\mathrm{AOS}$ is driven by the heat from the laser pulse and is therefore independent of the laser polarization and largely insensitive to any applied magnetic field [5,6]. The helicity-dependent AOS reported earlier could then be understood in terms of a differential light absorption induced by the magnetic circular dichroism in the magnetic alloy [7]. Finally, as these RE-TM alloys usually display chemical inhomogeneities, the potential role of superdiffusive spin currents has also being discussed [8].

While early studies concentrated on GdFeCo alloys, magnetization switching by laser pulses has now been reported in a

\footnotetext{
*1oic.le.guyader@xfel.eu; Present address: Spectroscopy, and Coherent Scattering, European XFEL GmbH, Notkestrasse 85, 22607 Hamburg, Germany.

${ }^{\dagger}$ Present address: College of Science and Technology, Nihon University, 24-1 Narashinodai 7-chome, Funabashi-shi, Chiba 2748501, Japan.

${ }^{\ddagger}$ Present address: Institute for Quantum Electronics, Physics Department, ETH Zurich, CH-8093 Zurich, Switzerland.
}

growing range of systems, namely other RE-TM alloys [9] and multilayers [10], RE-free synthetic ferrimagnets [10,11], and granular ferromagnets [12]. These recent developments are raising a number of crucial questions for the understanding of the AOS phenomenon and its transfer to real-world applications. Among these, the exact role played by the magnetization compensation temperature $T_{\mathrm{M}}$ at which the magnetizations of the two sublattices cancel each other out remains a puzzle. On the one hand, strong changes in the magnetization dynamics upon crossing $T_{\mathrm{M}}$ have been observed [13-15], and AOS seems to occur preferably for alloys displaying a $T_{\mathrm{M}}$ that can be reached through laser excitation $[9,10,16]$. On the other hand, atomistic simulations of the spin dynamics as well as experiments have shown that AOS is feasible below and above $T_{\mathrm{M}}$ [6]. In addition, helicity-dependent magnetization switching in granular ferromagnets where no $T_{\mathrm{M}}$ exists has been reported [12]. Finally, in view of potential applications, it is crucial to be able to characterize to which extent AOS is a deterministic process.

In this article, we investigate all-optical magnetization switching in GdFe-based alloys using photoemission electron microscopy (PEEM) with x-ray magnetic circular dichroism (XMCD) as a contrast mechanism, allowing imaging of the magnetic domain configuration with a spatial resolution of approximately $100 \mathrm{~nm}$. Single linearly polarized laser pulses were used to excite a multidomain configuration at temperatures below and above the magnetization compensation temperature $T_{\mathrm{M}}$ of the alloys. Introducing the Pearson productmoment correlation coefficient on a series of XMCD images allows us to report an almost purely deterministic AOS in both cases. Extrinsic pulse-to-pulse laser pointing stability and intrinsic finite domain sizes and thermally activated domainwall motion are found to be the main limiting factors for purely deterministic AOS. Using time-resolved XMCD PEEM imaging of the magnetization dynamics upon femtosecond laser excitation with $70 \mathrm{ps}$ time resolution and approximately $200 \mathrm{~nm}$ spatial resolution, it is found that AOS can even be achieved against a $180 \mathrm{mT}$ applied magnetic field. Finally, 
a strong reduction of the switching window above $T_{\mathrm{M}}$ is observed that could be related in part with the proximity of the Curie temperature $T_{\mathrm{C}}$ of the sample.

\section{METHODS}

\section{A. Time-resolved XMCD PEEM}

To resolve the magnetic domain configuration and its dynamics upon AOS, the Elmitec photoemission electron microscope (PEEM) at the Surface/Interface: Microscopy (SIM) beamline [17] at the Swiss Light Source (SLS) was used. Employing the X-ray magnetic circular dichroism (XMCD) effect at the $\mathrm{Fe} L_{3}$ edge at $708 \mathrm{eV}$, a quantitative static determination of the Fe spin orientation with a $100 \mathrm{~nm}$ spatial resolution is possible [18]. From two images recorded with opposite x-ray helicity, an asymmetry image is computed that contains only normalized magnetic contrast information. Such an image typically shows white or black regions corresponding to magnetic domains with magnetizations of opposite directions with respect to the X-ray propagation vector [19]. Time-resolved measurements of the sample magnetization were performed by taking advantage of the pulsed nature of the X-rays produced by the SLS via the gating of the detection in synchronization to an isolated $\mathrm{x}$-ray pulse, reducing the acquired signal intensity by a factor of 100 . As the isolated $\mathrm{X}$-ray pulse is also more intense than the other pulses, the spatial resolution of the PEEM worsens due to the space-charge effect [20]. This scheme, presented in detail in Ref. [21], allows stroboscopic pump-probe imaging of the sample with a time resolution determined by the $70 \mathrm{ps}$ full width at half-maximum (FWHM) temporal $\mathrm{x}$-ray pulse length. At this time scale, both TM and RE magnetizations are in equilibrium such that measuring the Fe sublattice is sufficient to characterize the sample magnetization orientation. The pump laser pulses were produced by an XL-500 oscillator from Femtolasers Produktions $\mathrm{GmbH}$, with a $\lambda=800 \mathrm{~nm}$ wavelength, a $\tau=$ $50 \mathrm{fs}$ pulse duration of $500 \mathrm{~nJ}$ per pulse, and a $5.2 \mathrm{MHz}$ repetition rate. The latter is then reduced by a Pockels cell in combination with a crossed polarizer to match the $1.04 \mathrm{MHz}$ repetition rate of the isolated $x$-ray probe pulses. The time overlap $(t=0)$ between the laser and the x-ray pulse is unambiguously determined to better than \pm 15 ps by the sudden space charging [22,23] induced by the laser pump pulse, which reduces significantly the amount of photoemitted electrons imaged by the microscope. Finally, the sample could be cooled down with a flow of liquid nitrogen, and the temperature was measured with a thermocouple attached to the sample holder.

\section{B. Samples}

The samples were grown on Si substrates to achieve fast cooling between laser pulses during $\mathrm{MHz}$ repetition rate pump-probe experiments [24]. A $3 \mathrm{~nm}$ thin $\mathrm{Si}_{3} \mathrm{~N}_{4}$ capping layer is used to prevent oxidation while still allowing for the low-energy secondary electron to escape into the vacuum and be imaged with the PEEM [25]. The magnetic layer in the samples is thin enough to ensure that no domain forms within the layer thickness. Three different samples have been used for this study, and their composition was adjusted to obtain squared hysteresis loops and $T_{\mathrm{M}}$ at the desired temperature.
For this, additional samples were grown on glass substrate, and temperature-dependent hysteresis loops were measured in transmission using the Faraday effect. From these we extracted the coercive field and the loop signal amplitude for each sample, as shown in Fig. 1(a). From the temperature dependence of the MOKE signal, the Curie temperature of these different films is found to be similar and can be estimated to be around $600 \mathrm{~K}$. From the divergence of the coercivity, the $T_{\mathrm{M}}$ was determined for each sample composition, as shown in Fig. 1(b), which can be approximated by a straight line over a limited composition range [26]. The first sample with structure $\operatorname{AlTi}(10 \mathrm{~nm}) / \mathrm{Si}_{3} \mathrm{~N}_{4}(5 \mathrm{~nm}) / \mathrm{Gd}_{25} \mathrm{Fe}_{65.6} \mathrm{Co}_{9.4}(20 \mathrm{~nm}) / \mathrm{Si}_{3} \mathrm{~N}_{4}(3$ $\mathrm{nm}$ ) has a $T_{\mathrm{M}}$ of $390 \mathrm{~K}$, as seen in the peak in the temperature dependence of the coercivity shown in Fig. 1(a). The two other samples are $\mathrm{GdFe}$ alloys of composition $\mathrm{Si}_{3} \mathrm{~N}_{4}(5 \mathrm{~nm}) /$ $\mathrm{Gd}_{20} \mathrm{Fe}_{80}(30 \mathrm{~nm}) / \mathrm{Si}_{3} \mathrm{~N}_{4}(3 \mathrm{~nm})$ with an extrapolated $T_{\mathrm{M}}$ below $10 \mathrm{~K}$ and $\mathrm{Si}_{3} \mathrm{~N}_{4}(5 \mathrm{~nm}) / \mathrm{Gd}_{24} \mathrm{Fe}_{76}(30 \mathrm{~nm}) / \mathrm{Si}_{3} \mathrm{~N}_{4}(3 \mathrm{~nm})$ with an extrapolated $T_{\mathrm{M}}$ above $500 \mathrm{~K}$. For the three samples, the out-of-plane hysteresis at low and high temperature is squared, as shown in Figs. 1(c)-1(e). In the rest of the paper, each sample is referred to by a reduced notation consisting of the Gd content, such as, for example, Gd25FeCo or Gd20Fe. When deposited on $\mathrm{Si}$ instead of glass substrate, we noted that the magnetization compensation temperature of the $\mathrm{Gd} 25 \mathrm{FeCo}$ film shifts by $130 \mathrm{~K}$ to a lower temperature.

\section{Laser excitation}

The linearly $p$-polarized laser pump pulses were focused on the sample at a grazing incidence of $16^{\circ}$. The laser spot size was measured by scanning a $5-\mu \mathrm{m}$-wide slit at the sample position in front of a photodiode measuring the transmitted light intensity, and it was found to be 30 by $100 \mu \mathrm{m}$ FWHM. To estimate the temperature profile created by the absorbed laser energy in the multilayer, the differential absorptance $d A$ was calculated using a matrix formalism of the light scattering at the different interfaces and the light propagation inside the layers based on Abeles's formulas [27], and the results are shown in Fig. 2(a). Neglecting any heat diffusion, the laser-induced temperature increase is simply given by $\Delta T=\mathcal{F} d A /\left(\rho C_{p}\right)$, where $\mathcal{F}$ is the incoming laser fluence, $\rho$ is the density, and $C_{p}$ is the heat capacity. The temperature increase for an incident fluence of $1 \mathrm{~mJ} \mathrm{~cm}^{-2}$ is shown in Fig. 2(a) by the dashed line. The material parameter values used for the calculation are listed in Table I. From this, the average temperature increase through the GdFeCo layer at the center of the laser spot is estimated to be $85 \mathrm{~K}$ in this case. The temperature profile over the PEEM field of view is shown in Fig. 2(b) and can be seen to be inhomogeneous. This means that the switching region at the center and the nonswitching region at the border should coexist in the PEEM images.

\section{RESULTS}

\section{A. Single laser pulse excitation}

In view of the potential applications, the question of the repeatability of AOS is essential. AOS was therefore studied on a multidomain configuration in which one laser pulse excites several different magnetic domains at once. The magnetic domain configuration before and after single linearly polarized 

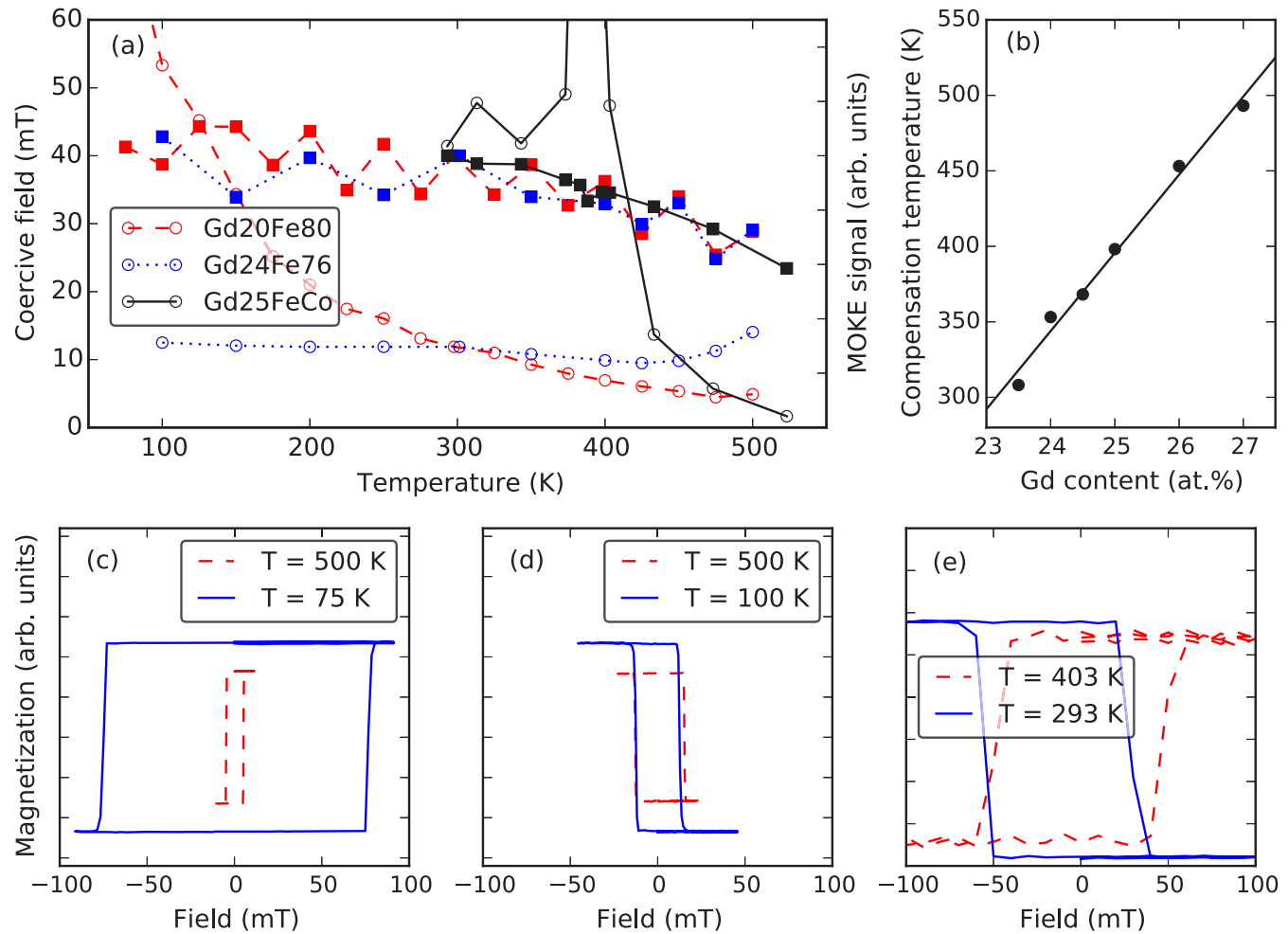

FIG. 1. (a) Temperature dependence of the coercivity (open circles) and MOKE signal amplitude (filled squares) of the three-sample system deposited on glass substrate. (b) Gd concentration dependence of the magnetization compensation temperature $T_{\mathrm{M}}$ around the Gd25FeCo system. The line is a linear fit while the points are the experimental data. Hysteresis loops at a low and high temperature for the (c) Gd18Fe, (d) Gd24Fe, and (e) Gd25FeCo sample system.

laser pulse exposure was recorded with static XMCD PEEM imaging. Sequences of such $I_{p}$ images [32] taken at the Fe $L_{3}$ edge for the $\mathrm{Gd} 25 \mathrm{FeCo}$ sample at a temperature above and below $T_{\mathrm{M}}$ in the absence of any applied magnetic field are shown in Figs. 3(a) and 3(c), respectively. In those images, white (black) contrast corresponds to magnetic domains whose out-of-plane magnetization has a positive (negative) projection on the x-ray direction, as indicated by the gray scale in Figs. 3(a) and 3(c). In both cases, below and above $T_{\mathrm{M}}$, changes in the magnetic domains in the center of the images are seen. To better emphasize the changes or the lack thereof occurring in these multidomain configurations, the pixel by pixel product between two successive images separated by a single linearly polarized laser pulse excitation $I_{p-1} I_{p}$ is computed [32] and shown in Figs. 3(b) and 3(d). Irrespective of the initial magnetic domain orientation, in the $I_{p-1} I_{p}$ image, a black contrast corresponds to a magnetization switching (SW), a gray contrast to a domain wall (DW), and a white contrast to an absence of changes, i.e., no switching (NS), as indicated by the gray scale in the inset. Visible in the product of successive images $I_{p-1} I_{p}$ shown in Figs. 3(b) and 3(d) is a black elongated elliptical region at the center surrounded by a white region unaffected by the laser pulses. This elongated elliptical shape corresponds to the laser spot size seen at the $16^{\circ}$ grazing incidence used in this experiment, as shown in Fig. 2(b). This black elongated region clearly corresponds to a laser-induced switching occurring equally for both magnetic domain orientations enclosed in the laser spot. Since this
AOS seems to occur with every laser pulse, it appears to be purely deterministic. To better quantify how deterministic this phenomenon of AOS really is, we introduce the pixel by pixel Pearson product-moment correlation coefficient $r$ [33] for a sequence of XMCD images as

$$
r=\frac{\sum_{p=1}^{n} I_{p-1} I_{p}}{\sqrt{\sum_{p=1}^{n} I_{p-1}^{2}} \sqrt{\sum_{p=1}^{n} I_{p}^{2}}},
$$

where $I_{p}$ is the XMCD image after $p$ laser pulses in the sequence. In the case of purely deterministic switching, this correlation coefficient $r$ is -1 , while in the absence of changes, i.e., no switching, $r=+1$. In the event of an unrelated domain configuration after every single laser pulse, such as in the case of heating above $T_{\mathrm{C}}, r=0$. Such correlation coefficient images $r$ calculated from the measured sequences are shown in Figs. 3(e) and 3(f) for a sample temperature above and below $T_{\mathrm{M}}$, respectively. The darkest region in these images corresponds indeed to a correlation coefficient of $r=-1$, i.e., a purely deterministic switching with each of the 10 laser pulses of the sequence, occurring both below and above $T_{\mathrm{M}}$. It is also evident that the spatial extent of this $r=-1$ region is limited by the spatial extent with which these 10 laser pulses overlap. Here in the particular case of this experiment, two effects are mainly responsible for this limited laser pulse overlap. First of all, with the laser being $6.5 \mathrm{~m}$ away from the PEEM, and the subsequent laser pulses being 
(a)

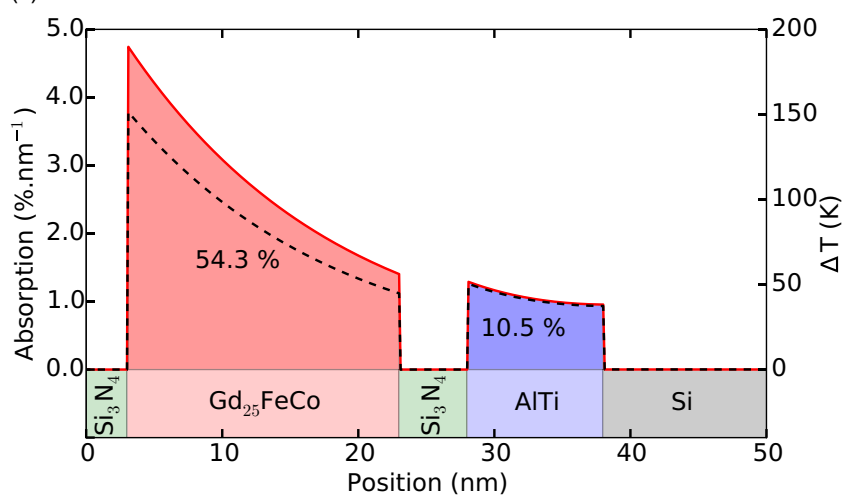

(b)

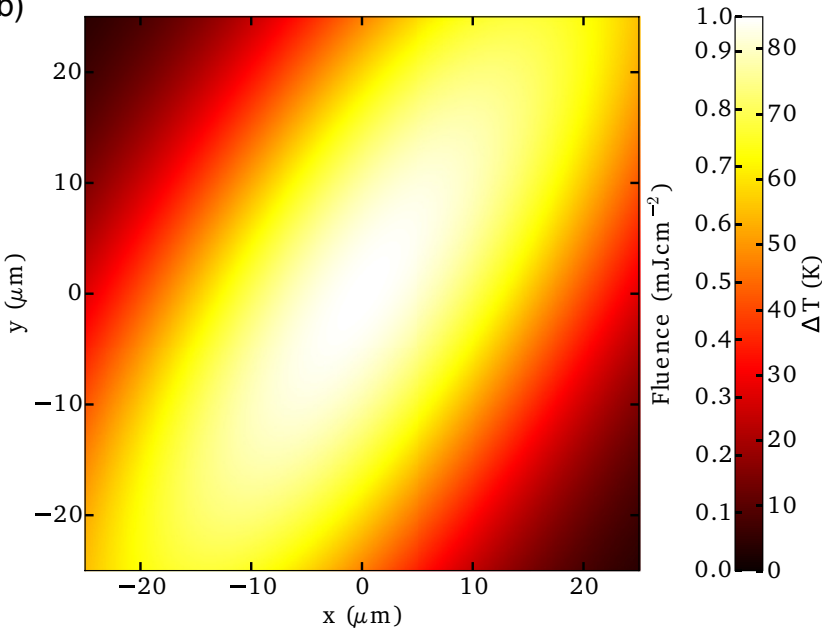

FIG. 2. (a) Calculated depth-resolved laser absorption (continuous line) in the $\mathrm{Gd} 25 \mathrm{FeCo}$ sample and laser-induced temperature increase (dashed line) in the case of an incoming laser fluence of $1 \mathrm{~mJ} \mathrm{~cm} \mathrm{~cm}^{-2}$. (b) Calculated spatially resolved laser fluence and temperature increase in the field of view imaged by the PEEM microscope for an incoming fluence of $1 \mathrm{~mJ} \mathrm{~cm}^{-2}$ from the measured laser spot size.

a minute apart, the laser shot-to-shot stability is inherently affected by air turbulence and thermal drifts in the optics. Secondly, as we vary the sample temperature, the PEEM sample manipulator contracts over the course of several hours,

TABLE I. The material parameters used in the calculation of the light absorption and temperature increase are the density $\rho$, heat capacity $C_{p}$ from Ref. [28] for $\mathrm{Si}_{3} \mathrm{~N}_{4}$, and from Ref. [29] for the other materials. The complex refractive index $\tilde{n}$ at $\lambda=800 \mathrm{~nm}$ wavelength is from Ref. [30] for GdFeCo and from Ref. [31] for the other materials. The values for AlTi and GdFeCo are the weighted average of the element constituents.

\begin{tabular}{lccc}
\hline \hline & $\rho$ & $C_{p}$ & \\
& $\left(10^{3} \mathrm{~kg} \mathrm{~m}^{-3}\right)$ & $\left(\mathrm{J} \mathrm{kg}^{-1} \mathrm{~K}^{-1}\right)$ & $\tilde{n}$ \\
\hline $\mathrm{Si}_{3} \mathrm{~N}_{4}$ & 3.17 & 711.3 & $2.0+0.0 i$ \\
$\mathrm{GdFeCo}$ & 7.97 & 393.1 & $3.7+3.856 i$ \\
$\mathrm{AlTi}$ & 3.60 & 710.0 & $2.81+5.89 i$ \\
$\mathrm{Si}$ & 2.329 & 712.0 & $3.68+0.005 i$ \\
\hline \hline
\end{tabular}

resulting in the apparent horizontal drift of the laser on the sample. Therefore, the pulse to pulse pointing stability is only an extrinsic limitation to a somewhat purely deterministic AOS.

However, there can also be intrinsic limitations such as that given by the domain walls, in particular at the boundary between the switching and nonswitching region of each laser pulse. For example, in the case of the sample temperature above $T_{\mathrm{M}}$ shown in Fig. 3(b), the domain wall at the bottom of the laser pulse region is almost continuously moving in the same direction between successive images, as indicated by the red arrows as well as the dashed ellipse in Fig. 3(e). As this domain wall is clearly outside the elongated elliptical region where AOS occurs, we know that the laser fluence is too low to induce a deterministic AOS. In fact, in the XMCD PEEM images $I_{1}$ and $I_{10}$ shown in Fig. 3(a), one can even see the domain-wall motion occurring during the imaging, which results in an extended gray region rather than either a completely black or completely white region. This is indicative of a very low coercivity of the domain walls at this temperature, which favors thermally activated domain-wall movements in the otherwise nonswitching region and should be regarded as intrinsically limiting the repeatability of the AOS. Comparing the domain size above and below $T_{\mathrm{M}}$, as shown in Figs. 3(a) and $3(\mathrm{c})$, one can immediately realize that the coercivity is higher in the second case as the magnetic domains are smaller, and thus more stable, in agreement with the temperature dependence of the coercivity shown in Fig. 1(a). Nevertheless, here some changes in the domain configuration can also be seen at the edges of the AOS region, as indicated by the blue arrow in the $I_{9} I_{10}$ image shown in Fig. 3(d). The small protuberance corresponds to a small black domain outside the AOS region, which disappeared between the images $I_{9}$ and $I_{10}$ shown in Fig. 3(c). This is likely the collapse of a too small domain formed by the intersection of the existent domain pattern and the AOS region created by the laser pulse. These processes of domain collapse and thermally activated domain-wall hopping should not be confused with AOS. In fact, they lower the repeatability of AOS.

Inside the $r=-1$ region, all magnetic domains are switching with every laser pulse. However, it is unclear what is happening for the domain wall separating them since the correlation coefficient $r$ is undefined there. To visualize the various domain-wall positions during the sequence of laser pulses, it is best to look at the low-intensity part of the squared image $I_{p}^{2}$ images [32], of which the sequence average $\left\langle I_{p}^{2}\right\rangle$ is shown in Figs. 3(g) and 3(h) for the sample temperature above and below $T_{\mathrm{M}}$, respectively. In those $\left\langle I_{p}^{2}\right\rangle$ images, the darker the domain wall, the less it moved during the sequence of laser pulses. In the case of the sample at a temperature above $T_{\mathrm{M}}$ shown in Fig. 3(g), some changes are visible at the domain wall inside the switching region, as indicated by the red arrow. In the case below $T_{\mathrm{M}}$ shown in Fig. 3(h), no changes are visible, meaning that the domain wall stayed in place within the $100 \mathrm{~nm}$ spatial resolution of the instrument. Considering the low coercivity of this material, this is a rather surprising and noteworthy feature of AOS. Nevertheless, evidence for potential domain-wall hopping well inside the $r=-1$ region is seen at least in one case, limiting the repeatability of the AOS. Overall, apart from the difference in coercivity, 

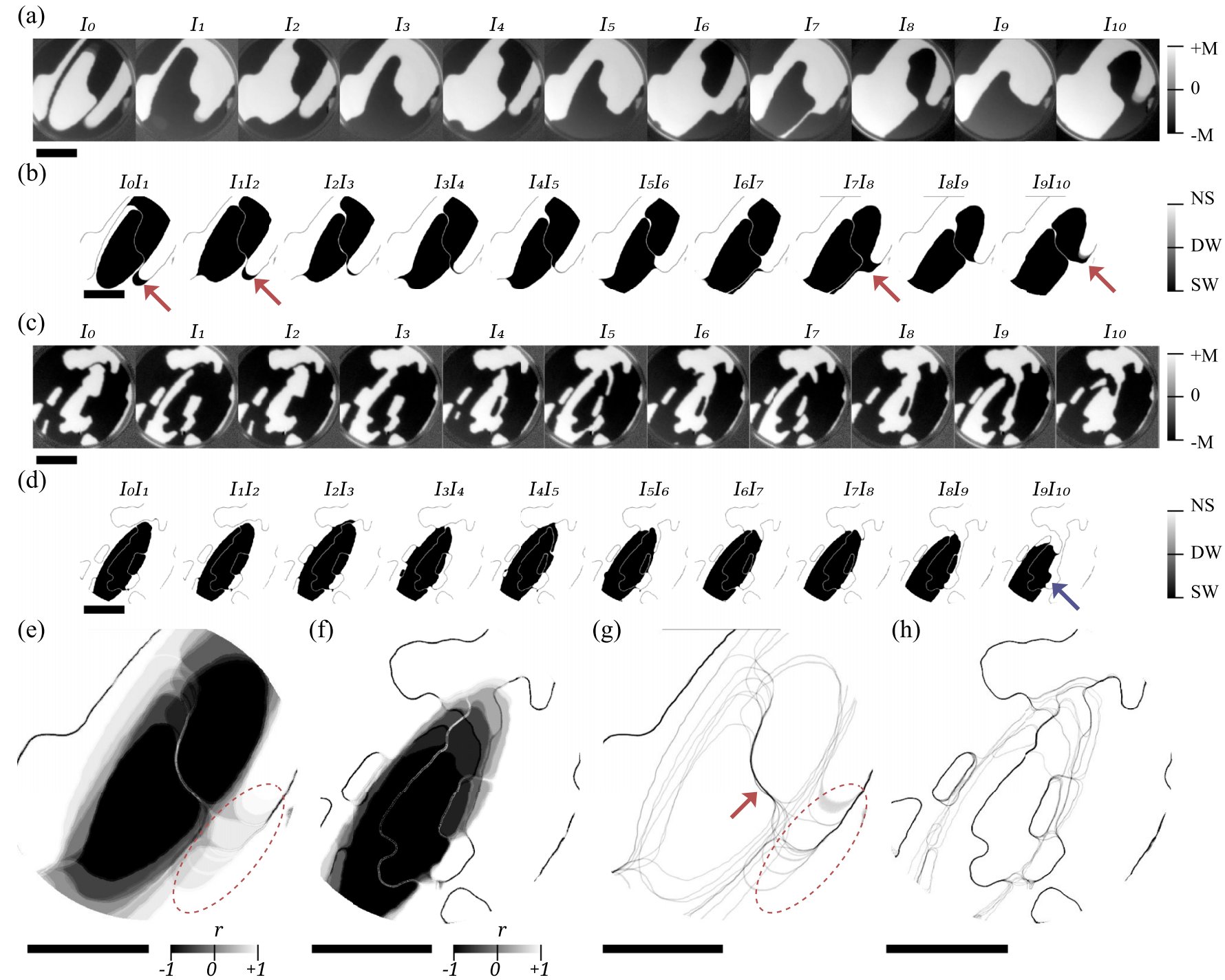

(g)

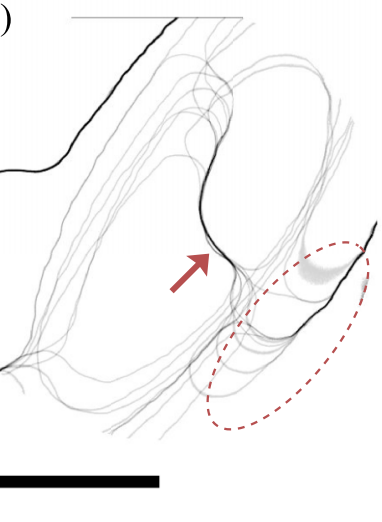

(h)

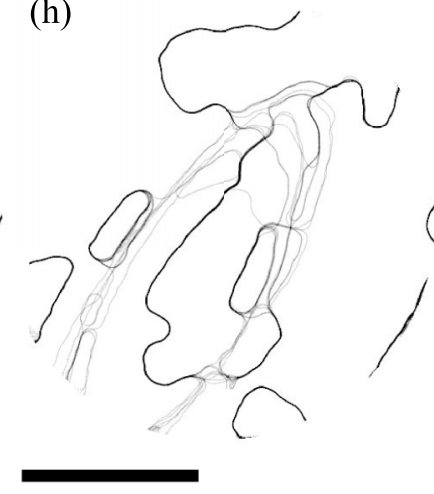

FIG. 3. Sequences of XMCD PEEM images $I_{p}$ taken after $p$ single laser pulse excitation (a) above at $T=300 \mathrm{~K}$ and (c) below at $T=160 \mathrm{~K}$; the magnetization compensation temperature $T_{\mathrm{M}}=260 \mathrm{~K}$ of the Gd25FeCo sample. The gray scale in the inset on the right indicates the out-of-plane magnetization orientation. Sequences of image product $I_{p-1} I_{p}$ (b) above and (d) below $T_{\mathrm{M}}$. The gray scale in the inset on the right indicates which gray level corresponds to magnetization switching (SW), no switching (NS), or domain wall (DW). Correlation coefficient images $r$ derived from the sequences of single laser pulse excitation (e) above and (f) below $T_{\mathrm{M}}$. Average image $\left\langle I_{p}^{2}\right\rangle$ showing the domain-wall positions $(\mathrm{g})$ above and $(\mathrm{h})$ below $T_{\mathrm{M}}$. Arrows and dashed ellipses indicate magnetization switching not related to AOS, as is discussed in the text. All scale bars are $20 \mu \mathrm{m}$.

very few differences are seen between AOS below and above $T_{\mathrm{M}}$.

\section{B. Time-resolved dynamics around $\boldsymbol{T}_{M}$}

To gain more insight into the AOS, and in particular into the role played by $T_{\mathrm{M}}$, the magnetization dynamics in this sample was investigated around $T_{\mathrm{M}}$. For this, time-resolved XMCD PEEM measurements were performed, and the results are shown in Fig. 4 for a sample temperature (a) above and (c) below $T_{\mathrm{M}}$, and for a strong $(H=180 \mathrm{mT})$ and a weak $(H=30$ $\mathrm{mT}$ ) out-of-plane magnetic field. The magnetic field is used to reset the sample magnetization to a well-defined initial state, allowing for stroboscopic measurement of the dynamics. The first thing to notice is that at negative time delay $t$, i.e., before the laser pulse, the sample is saturated for both applied magnetic fields, in contrast to the multidomain configuration observed in its absence in Fig. 3. Moreover, it can be seen that the orientation of the Fe sublattice magnetization reverses between Fig. 4(a) measured above $T_{\mathrm{M}}$ and Fig. 4(c) measured below $T_{\mathrm{M}}$, meaning that the sample is effectively on either side of the magnetization compensation temperature. Finally, while the PEEM lens settings were the same, the strong $180 \mathrm{mT}$ magnetic field can be seen to rotate the field of view and to zoom in by a factor of 1.5 compared with the $30 \mathrm{mT}$ case. From the timeresolved XMCD images, the magnetization dynamics at the center can be extracted, and it is shown in Figs. 4(b) and 4(d) for a sample temperature above and below $T_{\mathrm{M}}$, respectively. In both cases, magnetization switching occurs right after the laser pulse excites the sample. Thus, within the 70 ps time resolution 
(a)

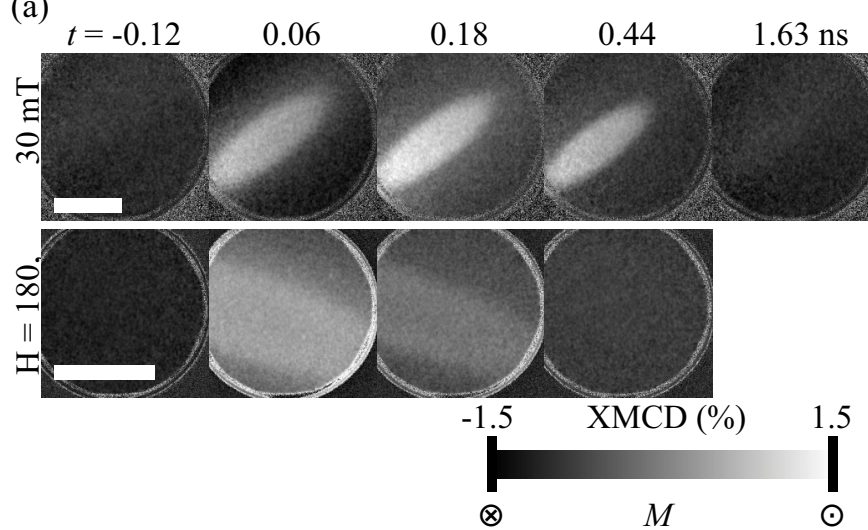

(b)

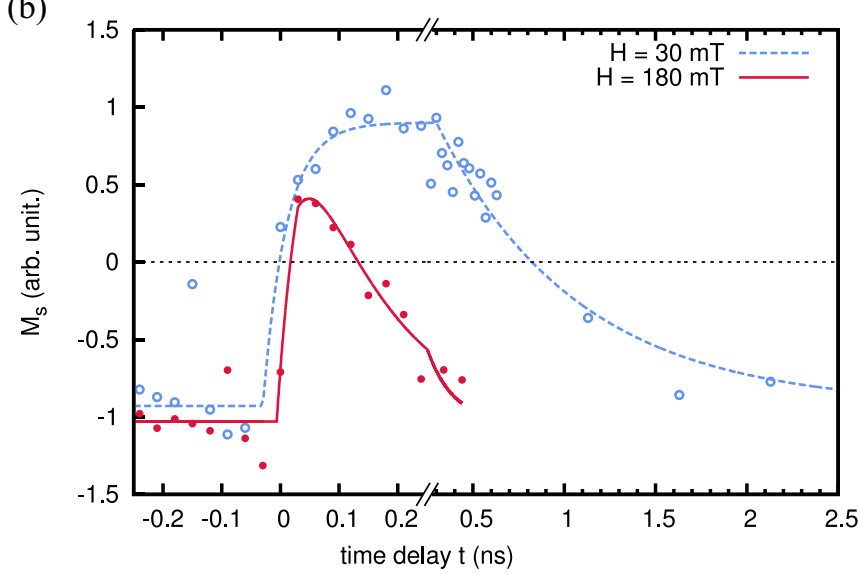

(c)

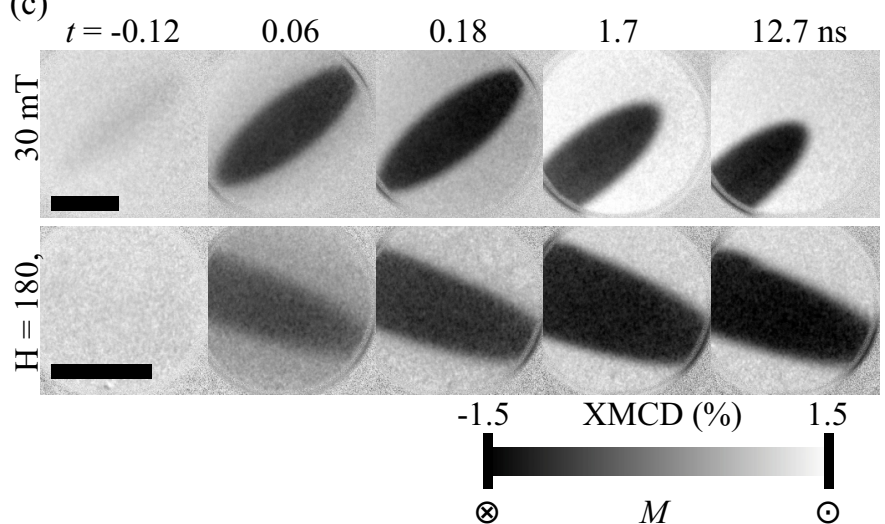

(d)

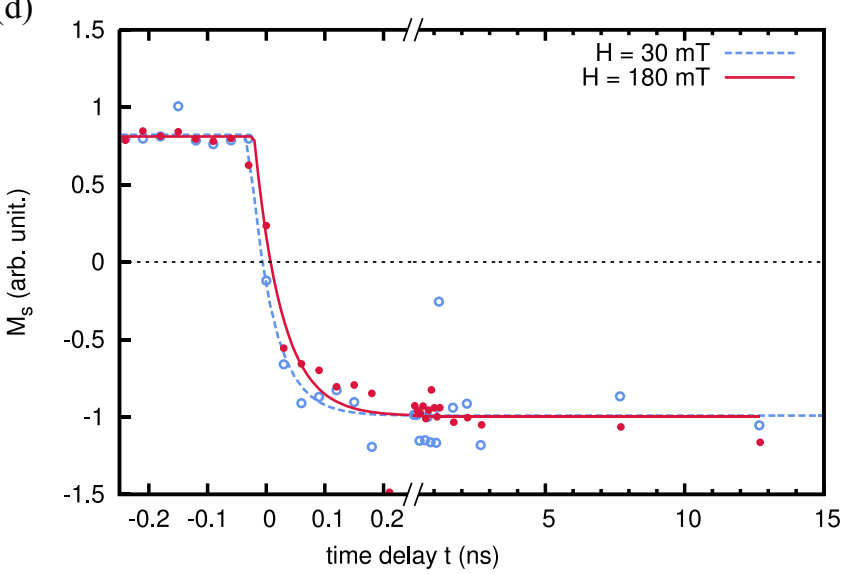

FIG. 4. (a) Time-resolved XMCD PEEM images on the Gd25FeCo sample at different time delays for two different applied out-of-plane magnetic fields of 30 and $180 \mathrm{mT}$, measured at the Fe $L_{3}$ edge, at a temperature above $T_{\mathrm{M}}$ at $T=300 \mathrm{~K}$, and (b) the extracted magnetization dynamics for each applied out-of-plane magnetic field. (c) and (d) Same as (a) and (b) for a sample temperature below $T_{\mathrm{M}}$ at $T=160 \mathrm{~K}$. The scale bars are $20 \mu \mathrm{m}$.

of the experiments, no difference is seen in the switching dynamics for either low or high magnetic field and either below or above $T_{\mathrm{M}}$. On the other hand, the relaxation toward the final state is strongly influenced by both the applied magnetic field and the sample base temperature. At a temperature above $T_{\mathrm{M}}$, as shown in Fig. 4(b), the reversed state is unstable against the applied magnetic field, leading to a fast relaxation toward the initial state; the faster the relaxation, the higher the field. It is worth noting here that switching with a laser pulse against a field of $180 \mathrm{mT}$ is thus possible, even though the relaxation is very fast, demonstrating the impetuous by which this AOS occurs [6]. Due to this fast relaxation and the $70 \mathrm{ps}$ long x-ray probe pulse length, a saturated switched state is not observed. At temperatures below $T_{\mathrm{M}}$ as shown in Fig. 4(d), the reversed state is now stable within the illuminated area, indicating that the temperature in this region is now above $T_{\mathrm{M}}$. In this case, after the laser pulse, the applied magnetic field is now stabilizing the reversed domain, leading to a very long lifetime.

Time-resolved XMCD PEEM images taken at the same fixed time delay of $t=+230 \mathrm{ps}$ after the laser pulse on the same Gd25FeCo sample are shown in Figs. 5(a) (above $T_{\mathrm{M}}$ ) and 5(b) (below $T_{\mathrm{M}}$ ) as a function of the laser pump fluence. A small static out-of-plane magnetic field of $H=30 \mathrm{mT}$ was applied to reset the sample after switching. This $30 \mathrm{mT}$ magnetic field is small enough not to hinder the AOS at this time scale, as can been seen in Fig. 4(b). While below $T_{\mathrm{M}}$ the laser fluence can be increased significantly without losing the AOS, the same is not true above $T_{\mathrm{M}}$. There, a small $10 \%$ increase from 2.7 to $3.0 \mathrm{~mJ} \mathrm{~cm}^{-2}$ is enough to bring the central region of the laser spot into a demagnetized state. This effect is most striking at the fluence of $\mathcal{F}=3.5 \mathrm{~mJ} \mathrm{~cm}^{-2}$ in Fig. 5(a), where no switching, switched states, and demagnetized states are all visible at once, with the switched region forming a very thin $2-\mu \mathrm{m}$-wide ring located on the steep edge of the laser pulse where the intensity gradient is the most pronounced, as seen in Fig. 2(b). The AOS fluence switching window is thus reduced above $T_{\mathrm{M}}$, and this asymmetry of the switching window around $T_{\mathrm{M}}$ is consistent with the literature [13-15]. Part of this effect might be attributed to the proximity with the Curie temperature $T_{C}$.

\section{Time-resolved dynamics far from $T_{M}$}

Due to the limited accessible temperature range in the PEEM, investigation of the AOS far from $T_{\mathrm{M}}$ requires samples with different compositions. For this, time-resolved XMCD PEEM measurement were thus performed on $\mathrm{Gd} 20 \mathrm{Fe}$ with $T_{\mathrm{M}}$ around $0 \mathrm{~K}$ and $\mathrm{Gd} 24 \mathrm{Fe}$ with $T_{\mathrm{M}}$ around $500 \mathrm{~K}$, under a small static out-of-plane magnetic field of $30 \mathrm{mT}$. The results are shown in Fig. 6. For both samples, a time resolution 
(a)

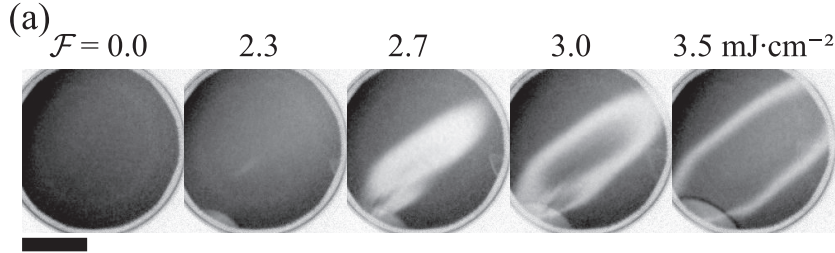

(b)

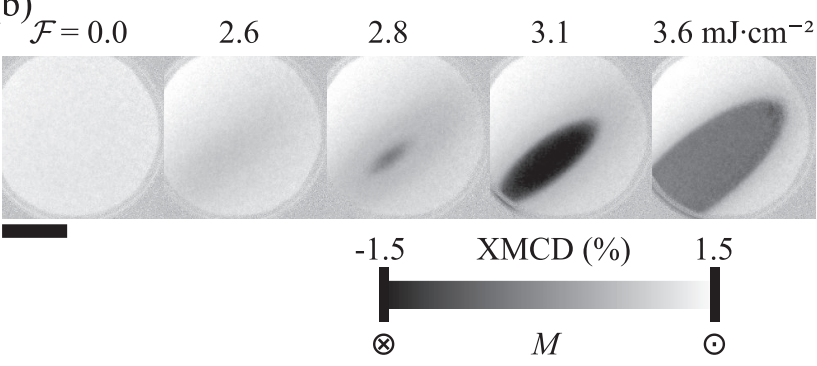

FIG. 5. Time-resolved XMCD PEEM images taken at $t=230$ ps after the laser pulse on the Gd25FeCo sample (a) above at $T=$ $300 \mathrm{~K}$ and (b) below at $T=160 \mathrm{~K}$ (the magnetization compensation temperature $T_{\mathrm{M}}=260 \mathrm{~K}$ ), as a function of the laser pump fluence. The static out-of-plane magnetic field was $30 \mathrm{mT}$. The scale bars are $20 \mu \mathrm{m}$.

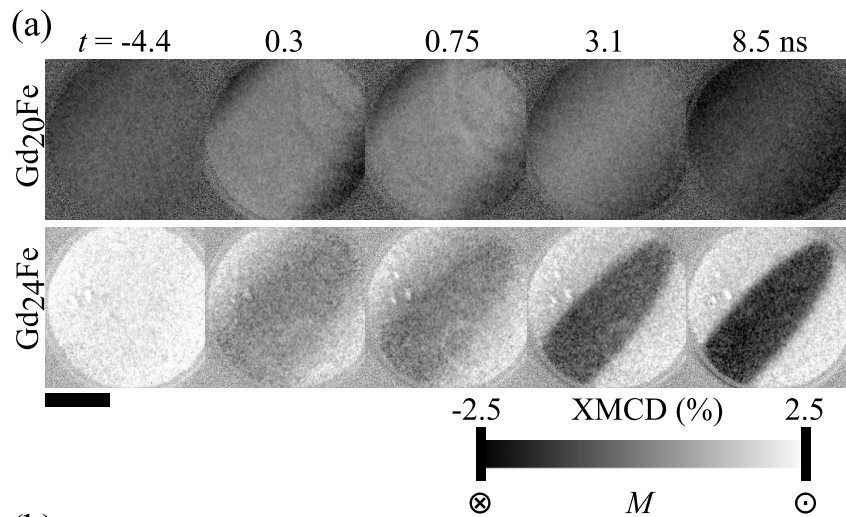

(b)

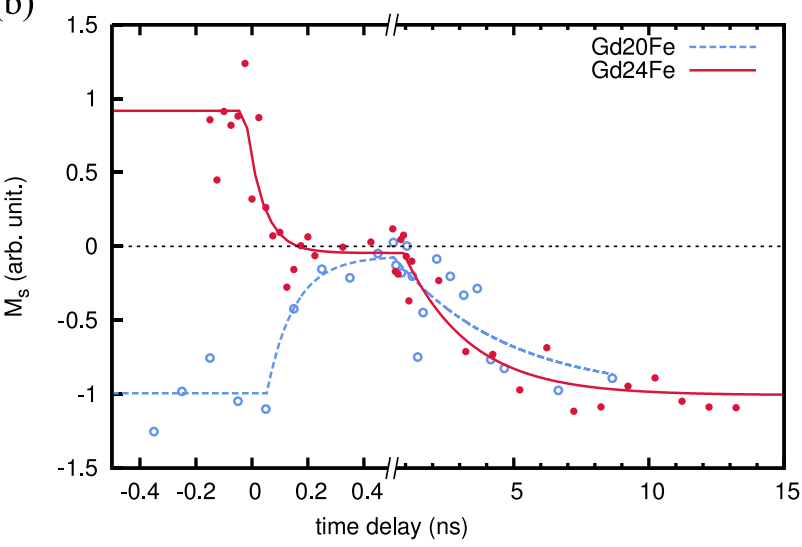

FIG. 6. (a) Time-resolved XMCD PEEM images at various fixed time delays, and (b) extracted magnetization dynamics on $\mathrm{Gd} 20 \mathrm{Fe}$ $\left(T_{\mathrm{M}}\right.$ around $\left.0 \mathrm{~K}, \mathcal{F}=5.7 \mathrm{~mJ} \mathrm{~cm}^{-2}\right)$ and $\mathrm{Gd} 24 \mathrm{Fe}\left(T_{\mathrm{M}}\right.$ around $500 \mathrm{~K}$, $\mathcal{F}=3.9 \mathrm{~mJ} \mathrm{~cm}^{-2}$ ) samples at room temperature with a $30 \mathrm{mT}$ outof-plane magnetic field. The scale bar is $20 \mu \mathrm{m}$. limited demagnetization process occurs. The samples then stay demagnetized for about $500 \mathrm{ps}$, which is then followed by a slow dynamics on a time scale of around $10 \mathrm{~ns}$, toward the initial state for $\mathrm{Gd} 20 \mathrm{Fe}$ and toward the reversed state for $\mathrm{Gd} 24 \mathrm{Fe}$. This reversal in the $\mathrm{Gd} 24 \mathrm{Fe}$ sample shows that there is an accessible magnetization compensation temperature $T_{\mathrm{M}}$ in this sample below $T_{\mathrm{C}}$, allowing the applied $30 \mathrm{mT}$ out-of-plane magnetic field to reverse the sample magnetization on a slow few-nanoseconds-long time scale and eventually back to the initial state at an even longer time scale after cooling down. For the $\mathrm{Gd} 20 \mathrm{Fe}$ sample, the temperature is already above $T_{\mathrm{M}}$ before the laser pulse, and therefore no magnetic field assisted switching occurs. Looking at the XMCD PEEM images taken at fixed time delay and shown in Fig. 6(a), it can be seen that in the case of the $\mathrm{Gd} 20 \mathrm{Fe}$ sample, the demagnetized region has a diffuse boundary, meaning that no magnetic domain is actually formed. On the other hand, for $\mathrm{Gd} 24 \mathrm{Fe}$, at around $750 \mathrm{ps}$ after the laser pulse, a clear boundary appears in the heated region, which is seen in Fig. 6(a) at $t=3.1 \mathrm{~ns}$. This very late formation of the reversed domain in $\mathrm{Gd} 24 \mathrm{Fe}$ and the absence of switching in $\mathrm{Gd} 20 \mathrm{Fe}$ allow us to conclude that no AOS window exists far from $T_{\mathrm{M}}$.

\section{DISCUSSION}

Determining if a system can display all-optical magnetization switching and to which extent this AOS is deterministic are two questions of crucial importance for a better understanding of the phenomenon as well as in view of its potential applications. In this context, sequences of XMCD PEEM images separated by single linearly polarized laser pulse excitation on a multidomain configuration such as shown in Fig. 3 can provide valuable information. First of all, since linearly $p$-polarized laser pulses are equally absorbed by each domain orientation, a direct comparison between what happens inside each domain is possible [7]. This is in contrast with multiple circularly polarized laser pulses used in recent studies, such as in Refs. [10,12], where such a comparison can only be made after carefully taking into account the magnetic circular dichroism of the material. Second, randomly demagnetized initial states are better than saturated or artificially created domain states since no stray field is created that could influence the switching. Third, the reversed domain configuration in such a case is known to be stable as well, therefore a collapse of the reversed domain state because of too low coercivity or too high net magnetization is not to be expected [34]. Finally, from such a sequence of images, the actual reproducibility of AOS can be measured using the Pearson product-moment correlation coefficient $r$ as introduced.

From our analysis, it follows that the purely deterministic AOS observed in the GdFeCo samples is limited by a number of extrinsic and intrinsic effects. The largest limitation we observe in Figs. 3(e) and 3(f) is the pulse-to-pulse laser pointing stability, which is extrinsic in nature to the switching phenomenon itself. The second limitation observed is related to the stability of the domain configuration. For example, at the edge of the laser pulse, the overlap of the $r=-1$ switching region with the preexistent domain configuration can create domains that are too small to be stable, as seen 
in Fig. 3(d), $I_{9} I_{10}$. In addition, thermal activation of domain walls can occur outside as well as inside the $r=-1$ switching region, as seen in Fig. 3(g) and indicated by the arrow and dashed ellipse. Since these two effects are related to the coercivity of the material, this constitutes an intrinsic limitation to the repeatability of the AOS. However, by understanding these limitations, we can envisage that engineered materials can potentially alleviate these limitations. For example, in patterned materials where each structure preferably hosts a single magnetic domain, a purely deterministic switching would be maintained.

Regarding the role played by the magnetization compensation temperature $T_{\mathrm{M}}$ on the AOS, we first confirm previous studies showing that AOS occurs below and above $T_{\mathrm{M}}[6,14]$. Single-shot laser pulse experiments shown in Fig. 3 as well as time-resolved measurements of the magnetization dynamics shown in Fig. 4 both reveal AOS below as well as above $T_{\mathrm{M}}$. However, there exists a clear difference between switching below and above $T_{\mathrm{M}}$, as shown in the fluence-dependent patterns observed at $t=230 \mathrm{ps}$ in Fig. 5. In addition, for $\mathrm{GdFe}$ samples with no $T_{\mathrm{M}}$, no switching is observed, as shown in Fig. 6. This leads to the conclusion that while the existence of a reachable $T_{\mathrm{M}}$ during laser excitation is not a strict requirement to observe AOS, sample compositions with $T_{\mathrm{M}}$ near room temperature are preferred. It must be noted that in addition to $T_{\mathrm{M}}$, an angular momentum compensation temperature $T_{\mathrm{A}}$ also exists at a slightly higher temperature [35]. However, our experimental geometry with an out-of-plane magnetic field does not enable magnetization precession dynamics to be observed, precluding any investigation of the effect of $T_{\mathrm{A}}$ on AOS. Finally, AOS is a very robust switching mechanism as it can be realized against an opposing applied magnetic field [6], as demonstrated experimentally here in the case of a $180 \mathrm{mT}$ field in Fig. 4(b).

\section{CONCLUSIONS}

In conclusion, using static and time-resolved PEEM microscopy with XMCD to probe the sample magnetization upon laser excitation, important aspects of the AOS have been revealed. Sequences of images after single linearly polarized laser pulse excitation on a multidomain configuration allow for the study of the repeatability of the process by using the correlation coefficient as its measure. It is found that the AOS in the $\mathrm{Gd} 25 \mathrm{FeCo}$ sample studied is almost purely deterministic. Moreover, the intrinsic limitation from the low coercivity of the material leading to thermally activated domain-wall hopping could be alleviated in patterned media. From the time-resolved measurement of the magnetization dynamics, it is found that AOS occurs below and above $T_{\mathrm{M}}$, while on the other hand, no AOS occurs for sample temperatures far from it. A strong reduction of the fluence switching window occurs above $T_{\mathrm{M}}$ and is likely related to its proximity to the Curie temperature $T_{\mathrm{C}}$. Finally, AOS against an applied magnetic field of $180 \mathrm{mT}$ is demonstrated, illustrating the impetus by which AOS occurs.

\section{ACKNOWLEDGMENTS}

We thank the European Research Council under the European Union's Seventh Framework Programme FP7/20072013 [Grants No. NMP3-SL-2008-214469 (UltraMagnetron), FP7-NMP-2011-SMALL- No. 281043 (FEMTOSPIN), No. 214810 (FANTOMAS), and No. 339813 (Exchange)] for part of the financial supports as well as the MEXT-Supported Program for the Strategic Research Foundation at Private Universities, 2013-2017. Part of this work was performed at the Swiss Light Source, Paul Scherrer Institut, Villigen, Switzerland. We thank J. Honegger for his support.
[1] J. Stöhr and H. C. Siegmann, Magnetism: From Fundamentals to Nanoscale Dynamics (Springer-Verlag, Berlin, 2006).

[2] E. Beaurepaire, J.-C. Merle, A. Daunois, and J.-Y. Bigot, Ultrafast Spin Dynamics in Ferromagnetic Nickel, Phys. Rev. Lett. 76, 4250 (1996).

[3] A. Kirilyuk, A. V. Kimel, and Th. Rasing, Ultrafast optical manipulation of magnetic order, Rev. Mod. Phys. 82, 2731 (2010).

[4] C. D. Stanciu, F. Hansteen, A. V. Kimel, A. Kirilyuk, A. Tsukamoto, A. Itoh, and Th. Rasing, All-Optical Magnetic Recording with Circularly Polarized Light, Phys. Rev. Lett. 99, 047601 (2007).

[5] I. Radu, K. Vahaplar, C. Stamm, T. Kachel, N. Pontius, H. A. Dürr, T. A. Ostler, J. Barker, R. F. L. Evans, R. W. Chantrell, A. Tsukamoto, A. Itoh, A. Kirilyuk, Th. Rasing, and A. V. Kimel, Transient ferromagnetic-like state mediating ultrafast reversal of antiferromagnetically coupled spins, Nature (London) 472, 205 (2011)

[6] T. A. Ostler, J. Barker, R. F. L. Evans, R. Chantrell, U. Atxitia, O. Chubykalo-Fesenko, S. El Moussaoui, L. Le Guyader, E.
Mengotti, L. J. Heyderman, F. Nolting, A. Tsukamoto, A. Itoh, D. Afanasiev, B. A. Ivanov et al., Ultrafast heating as a sufficient stimulus for magnetization reversal in a ferrimagnet, Nat. Commun. 3, 666 (2012).

[7] A. R. Khorsand, M. Savoini, A. Kirilyuk, A. V. Kimel, A. Tsukamoto, A. Itoh, and Th. Rasing, Role of Magnetic Circular Dichroism in All-Optical Magnetic Recording, Phys. Rev. Lett. 108, 127205 (2012).

[8] C. E. Graves, A. H. Reid, T. Wang, B. Wu, S. de Jong, K. Vahaplar, I. Radu, D. P. Bernstein, M. Messerschmidt, L. Müller, R. Coffee, M. Bionta, S. W. Epp, R. Hartmann, N. Kimmel et al., Nanoscale spin reversal by non-local angular momentum transfer following ultrafast laser excitation in ferrimagnetic GdFeCo, Nat. Mater. 12, 293 (2013).

[9] S. Alebrand, M. Gottwald, M. Hehn, D. Steil, M. Cinchetti, D. Lacour, E. E. Fullerton, M. Aeschlimann, and S. Mangin, Light-induced magnetization reversal of high-anisotropy TbCo alloy films, Appl. Phys. Lett. 101, 162408 (2012).

[10] S. Mangin, M. Gottwald, C.-H. Lambert, D. Steil, V. Uhliř, L. Pang, M. Hehn, S. Alebrand, M. Cinchetti, G. Malinowski, 
Y. Fainman, M. Aeschlimann, and E. E. Fullerton, Engineered materials for all-optical helicity-dependent magnetic switching, Nat. Mater. 13, 286 (2014).

[11] R. F. L. Evans, Th. A. Ostler, R. W. Chantrell, I. Radu, and Th. Rasing, Ultrafast thermally induced magnetic switching in synthetic ferrimagnets, Appl. Phys. Lett. 104, 082410 (2014).

[12] C.-H. Lambert, S. Mangin, B. S. D. Ch. S. Varaprasad, Y. K. Takahashi, M. Hehn, M. Cinchetti, G. Malinowski, K. Hono, Y. Fainman, M. Aeschlimann, and E. E. Fullerton, All-optical control of ferromagnetic thin films and nanostructures, Science 345, 1337 (2014).

[13] K. Vahaplar, A. M. Kalashnikova, A. V. Kimel, D. Hinzke, U. Nowak, R. Chantrell, A. Tsukamoto, A. Itoh, A. Kirilyuk, and Th. Rasing, Ultrafast Path for Optical Magnetization Reversal via a Strongly Nonequilibrium State, Phys. Rev. Lett. 103, 117201 (2009).

[14] K. Vahaplar, A. M. Kalashnikova, A. V. Kimel, S. Gerlach, D. Hinzke, U. Nowak, R. Chantrell, A. Tsukamoto, A. Itoh, A. Kirilyuk, and Th. Rasing, All-optical magnetization reversal by circularly polarized laser pulses: Experiment and multiscale modeling, Phys. Rev. B 85, 104402 (2012).

[15] R. Medapalli, I. Razdolski, M. Savoini, A. R. Khorsand, A. Kirilyuk, A. V. Kimel, Th. Rasing, A. M. Kalashnikova, A. Tsukamoto, and A. Itoh, Efficiency of ultrafast laser-induced demagnetization in $\mathrm{Gd}_{x} \mathrm{Fe}_{100-x-y} \mathrm{Co}_{y}$ alloys, Phys. Rev. B 86, 054442 (2012).

[16] S. Alebrand, A. Hassdenteufel, D. Steil, M. Bader, A. Fischer, M. Cinchetti, and M. Aeschlimann, All-optical magnetization switching using phase shaped ultrashort laser pulses, Phys. Status Solidi A 209, 2589 (2012).

[17] U. Flechsig, F. Nolting, A. Fraile Rodríguez, J. Krempaský, C. Quitmann, T. Schmidt, S. Spielmann, and D. Zimoch, in Performance Measurements at the SLS SIM Beamline, AIP Conf. Proc. No. 1234 (AIP, New York, 2010), p. 319.

[18] F. Nolting, in Magnetic Imaging with X-rays, Magnetism and Synchrotron Radiation (Springer-Verlag, Berlin, 2010), p. 345.

[19] A. Scholl, H. Ohldag, F. Nolting, J. Stöhr, and H. A. Padmore, $\mathrm{X}$-ray photoemission electron microscopy, a tool for the investigation of complex magnetic structures (invited), Rev. Sci. Instrum. 73, 1362 (2002)

[20] Th. Schmidt, A. Sala, H. Marchetto, E. Umbach, and H.-J. Freund, First experimental proof for aberration correction in XPEEM: Resolution, transmission enhancement, and limitation by space charge effects, Ultramicroscopy 126, 23 (2013).

[21] L. Le Guyader, A. Kleibert, A. Fraile Rodríguez, S. El Moussaoui, A. Balan, M. Buzzi, J. Raabe, and F. Nolting, Studying nanomagnets and magnetic heterostructures with X-ray PEEM at the Swiss Light Source, J. Electron. Spectrosc. Relat. Phenom. 185, 371 (2012).
[22] A. Mikkelsen, J. Schwenke, T. Fordell, G. Luo, K. Klunder, E. Hilner, N. Anttu, A. A. Zakharov, E. Lundgren, J. Mauritsson, J. N. Andersen, H. Q. Xu, and A. L'Huillier, Photoemission electron microscopy using extreme ultraviolet attosecond pulse trains, Rev. Sci. Instrum. 80, 123703 (2009).

[23] N. M. Buckanie, J. Göhre, P. Zhou, D. von der Linde, M. Horn-von Hoegen, and F-J. Meyer zu Heringdorf, Space charge effects in photoemission electron microscopy using amplified femtosecond laser pulses, J. Phys. Condens. Matter 21, 314003 (2009).

[24] A. Hassdenteufel, Ch. Schubert, B. Hebler, H. Schultheiss, J. Fassbender, M. Albrecht, and R. Bratschitsch, All-optical helicity dependent magnetic switching in Tb-Fe thin films with a MHz laser oscillator, Opt. Express 22, 10017 (2014).

[25] J. Stöhr, H. A. Padmore, S. Anders, T. Stammler, and M. R. Scheinfein, Principles of x-ray magnetic dichroism spectromicroscopy, Surf. Rev. Lett. 05, 1297 (1998).

[26] T. A. Ostler, R. F. L. Evans, R. W. Chantrell, U. Atxitia, O. Chubykalo-Fesenko, I. Radu, R. Abrudan, F. Radu, A. Tsukamoto, A. Itoh, A. Kirilyuk, Th. Rasing, and A. Kimel, Crystallographically amorphous ferrimagnetic alloys: Comparing a localized atomistic spin model with experiments, Phys. Rev. B 84, 024407 (2011).

[27] K. Ohta and H. Ishida, Matrix formalism for calculation of the light beam intensity in stratified multilayered films, and its use in the analysis of emission spectra, Appl. Opt. 29, 2466 (1990).

[28] CRC Materials Science and Engineering Handbook, edited by J. F. Shackelford and W. Alexander (CRC, Boca Raton, FL, 2001).

[29] CRC Handbook of Chemistry and Physics, edited by W. M. Haynes and D. R. Lide (CRC, Boca Raton, FL, 2011).

[30] B. Koene, M. Savoini, A. V. Kimel, A. Kirilyuk, and Th. Rasing, Optical energy optimization at the nanoscale by near-field interference, Appl. Phys. Lett. 101, 013115 (2012).

[31] Handbook of Optical Constants of Solids I, II and III, edited by E. D. Palik (Academic, Burlington, VT, 1997).

[32] See Supplemental Material at http://link.aps.org/supplemental/ 10.1103/PhysRevB.93.134402 for the videos of the image sequences $I_{p}, I_{p-1} I_{p}$, and $I_{p}^{2}$ measured below and above the magnetization compensation temperature.

[33] K. Pearson, Notes on regression and inheritance in the case of two parents, Proc. R. Soc. 58, 240 (1895).

[34] C. Schubert, A. Hassdenteufel, P. Matthes, J. Schmidt, M. Helm, R. Bratschitsch, and M. Albrecht, All-optical helicity dependent magnetic switching in an artificial zero moment magnet, Appl. Phys. Lett. 104, 082406 (2014).

[35] C. D. Stanciu, A. V. Kimel, F. Hansteen, A. Tsukamoto, A. Itoh, A. Kiriliyuk, and Th. Rasing, Ultrafast spin dynamics across compensation points in ferrimagnetic GdFeCo: The role of angular momentum compensation, Phys. Rev. B 73, 220402 (2006). 